\title{
Transplantation operators and Cesàro operators for the Hankel transform
}

\author{
by \\ YUichi Kanjin (Kanazawa)
}

\begin{abstract}
The transplantation operators for the Hankel transform are considered. We prove that the transplantation operator maps an integrable function under certain conditions to an integrable function. As an application, we obtain the $L^{1}$-boundedness and $H^{1}$-boundedness of Cesàro operators for the Hankel transform.
\end{abstract}

1. Introduction and results. The Hankel transform $\mathcal{H}_{\mu} f$ of order $\mu$ of a function $f$ on the open half line $(0, \infty)$ is defined by

$$
\mathcal{H}_{\mu} f(y)=\int_{0}^{\infty} f(t) \sqrt{y t} J_{\mu}(y t) d t, \quad y>0
$$

where $J_{\mu}$ is the Bessel function of the first kind of order $\mu$. The Bessel functions with $\mu=-1 / 2$ and $\mu=1 / 2$ are $J_{-1 / 2}(z)=\sqrt{2 /(\pi z)} \cos z$ and $J_{1 / 2}(z)=\sqrt{2 /(\pi z)} \sin z$, and the Hankel transforms $\mathcal{H}_{-1 / 2} f$ and $\mathcal{H}_{1 / 2} f$ are the cosine and sine transforms:

$$
\mathcal{H}_{-1 / 2} f(y)=\sqrt{\frac{2}{\pi}} \int_{0}^{\infty} f(t) \cos y t d t, \quad \mathcal{H}_{1 / 2} f(y)=\sqrt{\frac{2}{\pi}} \int_{0}^{\infty} f(t) \sin y t d t .
$$

It is known that for $\mu \geq-1 / 2, \mathcal{H}_{\mu}$ is an isometry on $L^{2}(0, \infty)$ (Parseval's theorem for the Hankel transform) and $\mathcal{H}_{\mu} \mathcal{H}_{\mu}=I$ (the inversion formula for the Hankel transform), where $I$ is the identity operator and $L^{2}(0, \infty)$ is the Lebesgue space of functions on $(0, \infty)$ with $\|f\|_{2}=\left(\int_{0}^{\infty}|f(x)|^{2} d x\right)^{1 / 2}<\infty$. From now on, we let $\mu, \nu \geq-1 / 2$ unless otherwise stated explicitly. We put

$$
T f(x)=\frac{1}{x} \int_{0}^{x} f(y) d y, \quad S f(x)=\int_{x}^{\infty} f(y) \frac{d y}{y}, \quad x>0 .
$$

Then $T$ and $S$ are bounded operators on $L^{2}(0, \infty)$ because of the Hardy integral inequalities:

2000 Mathematics Subject Classification: Primary 42A38; Secondary 42A50, 42C10.

Key words and phrases: Cesàro operator, transplantation operator, Hankel transform.

Partly supported by the Grants-in-Aid for Scientific Research (C), Japan Society for the Promotion of Science. 


$$
\begin{aligned}
& \int_{0}^{\infty}|T f(x)|^{p} d x \leq\left(\frac{p}{p-1}\right)^{p} \int_{0}^{\infty}|f(x)|^{p} d x, \\
& \int_{0}^{\infty}|S f(x)|^{q} d x \leq q^{q} \int_{0}^{\infty}|f(x)|^{q} d x,
\end{aligned}
$$

where $1<p \leq \infty$ and $1 \leq q<\infty$ (cf. [3, Corollary 6.21]). For $f \in L^{2}(0, \infty)$, its Hankel transform $\mathcal{H}_{\nu} f$ of order $\nu$ is in $L^{2}(0, \infty)$, and so $T \mathcal{H}_{\nu} f \in L^{2}(0, \infty)$. Let us define the function $\mathcal{C}_{\mu}^{\nu} f \in L^{2}(0, \infty)$ so that its Hankel transform $\mathcal{H}_{\mu}\left(\mathcal{C}_{\mu}^{\nu} f\right)$ of order $\mu$ is $T \mathcal{H}_{\nu} f$, that is,

$$
\mathcal{H}_{\mu}\left(\mathcal{C}_{\mu}^{\nu} f\right)(x)=\frac{1}{x} \int_{0}^{x} \mathcal{H}_{\nu} f(t) d t, \quad x>0
$$

The objects of our study are the composition $\mathcal{T}_{\mu}^{\nu}=\mathcal{H}_{\mu} \mathcal{H}_{\nu}$ and the operator $\mathcal{C}_{\mu}^{\nu}$ initially defined on $L^{2}(0, \infty)$. We call $\mathcal{T}_{\mu}^{\nu}$ the transplantation operator for the Hankel transform from $\nu$ to $\mu$, and $\mathcal{C}_{\mu}^{\nu}$ the Cesàro operator for the Hankel transform with index $(\nu, \mu)$.

Let $H g$ be the Hilbert transform of a function $g$ on the line $(-\infty, \infty)$ :

$$
H g(x)=\lim _{\delta \rightarrow+0} \frac{1}{\pi} \int_{|x-t|>\delta} \frac{g(t)}{x-t} d t, \quad-\infty<x<\infty .
$$

We denote by $R g$ the restriction of $g$ to the half-open interval $(0, \infty): R g=$ $\left.g\right|_{(0, \infty)}$, and denote by $g_{\mathrm{e}}, g_{\mathrm{o}}$ the even and odd parts of $g$, that is, $g_{\mathrm{e}}(x)=$ $(g(x)+g(-x)) / 2, g_{\mathrm{o}}(x)=(g(x)-g(-x)) / 2$. We see that $H g_{\mathrm{e}}$ and $H g_{\mathrm{o}}$ are odd and even, respectively, and that for $g \in L^{2}(-\infty, \infty)$,

$$
H g(x)= \begin{cases}\mathcal{T}_{1 / 2}^{-1 / 2}\left(R g_{\mathrm{e}}\right)(x)+\mathcal{T}_{-1 / 2}^{1 / 2}\left(R g_{\mathrm{o}}\right)(x) & (\text { a.e. } x>0), \\ -\mathcal{T}_{1 / 2}^{-1 / 2}\left(R g_{\mathrm{e}}\right)(-x)+\mathcal{T}_{-1 / 2}^{1 / 2}\left(R g_{\mathrm{o}}\right)(-x) & (\text { a.e. } x<0),\end{cases}
$$

and in particular $H g_{\mathrm{e}}(x)=\mathcal{T}_{1 / 2}^{-1 / 2}\left(R g_{\mathrm{e}}\right)(x)$ and $H g_{\mathrm{o}}(x)=\mathcal{T}_{-1 / 2}^{1 / 2}\left(R g_{\mathrm{o}}\right)(x)$ for a.e. $x>0$. Therefore, the transplantation operators $\mathcal{T}_{\mu}^{\nu}$ are generalizations of the Hilbert transform $H$. Hardy [11] proved the following:

[A] (Hardy [11]). Suppose that

$$
\int_{-\infty}^{\infty}|g(x)| d x<\infty, \quad \int_{-\infty}^{\infty}|x||d g(x)|<\infty
$$

Then $H g_{\mathrm{o}}$ is integrable, and $H g_{\mathrm{e}}=(2 /(\pi|x|)) \int_{0}^{x} g_{\mathrm{e}}(t) d t+h$ with an integrable function $h$.

In this paper, we shall extend this result to all the transplantation operators $\mathcal{T}_{\mu}^{\nu}$, and as an application we shall obtain the $L^{1}$-boundedness and $H^{1}$-boundedness of the Cesàro operators $\mathcal{C}_{\mu}^{\nu}$. 
Historically, to get the Marcinkiewicz type multiplier theorem for the Hankel transform, Guy [9] proved that the operators $\mathcal{T}_{\mu}^{\nu}$, initially defined on $L^{2}(0, \infty)$, extend to bounded operators on the $L^{p}$-spaces, $1<p<\infty$. His result is called the transplantation theorem for the Hankel transform, and it is the first of the transplantation theorem for classical expansions. Schindler [23] showed a refined version of Guy's result by getting an explicit representation of $\mathcal{T}_{\mu}^{\nu}$. We recall it here for later convenience.

Let $T_{\mu, \nu}$ be the operator defined by

$$
\begin{gathered}
T_{\mu, \nu} f(x)=\lim _{\delta \rightarrow+0} \int_{|x-y|>\delta} f(y) \widetilde{I}_{\mu, \nu}(x, y) d y+k(\mu, \nu) f(x), \\
k(\mu, \nu)=\cos ((\mu-\nu) \pi / 2)
\end{gathered}
$$

where

$$
\begin{gathered}
\widetilde{I}_{\mu, \nu}(x, y)=K_{\mu, \nu} \sqrt{x y}\left(\frac{y}{x}\right)^{\nu} \frac{1}{x^{2}-y^{2}} F\left(\frac{\nu-\mu}{2}, \frac{\mu+\nu}{2} ; \nu+1 ; \frac{y^{2}}{x^{2}}\right) \\
=2^{-1} K_{\mu, \nu}\left(\frac{y}{x}\right)^{\nu+1 / 2}\left(\frac{1}{x-y}+\frac{1}{x+y}\right) F\left(\frac{\nu-\mu}{2}, \frac{\mu+\nu}{2} ; \nu+1 ; \frac{y^{2}}{x^{2}}\right), \\
K_{\mu, \nu}=\frac{2 \Gamma((\mu+\nu+2) / 2)}{\Gamma(\nu+1) \Gamma((\mu-\nu) / 2)}
\end{gathered}
$$

for $0<y<x$, and

$$
\widetilde{I}_{\mu, \nu}(x, y)=\widetilde{I}_{\nu, \mu}(y, x)
$$

for $y>x>0$. Here, $F(\alpha, \beta ; \gamma ; z)$ is the hypergeometric function, that is,

$$
F(\alpha, \beta ; \gamma ; z)=\sum_{k=0}^{\infty} \frac{(\alpha)_{k}(\beta)_{k}}{(\gamma)_{k} k !} z^{k}, \quad|z|<1,
$$

where $(\lambda)_{0}=1,(\lambda)_{k}=\lambda(\lambda+1) \cdots(\lambda+k-1), k \geq 1$.

[B] (Schindler [23]).

(i) For $f \in C_{\mathrm{c}}^{\infty}(0, \infty), \mathcal{T}_{\mu}^{\nu} f(x)=T_{\mu, \nu} f(x)$ a.e. $x>0$, where $C_{\mathrm{c}}^{\infty}(0, \infty)$ is the space of infinitely differentiable functions of compact support in $(0, \infty)$.

(ii) Let $1<p<\infty$ and $-1 / p<\alpha<1-1 / p$. If $\int_{0}^{\infty}|f(x)|^{p} x^{\alpha p} d x<\infty$, then the value $T_{\mu, \nu} f(x)$ exists for a.e. $x>0$, and

$$
\int_{0}^{\infty}\left|T_{\mu, \nu} f(x)\right|^{p} x^{\alpha p} d x \leq C \int_{0}^{\infty}|f(x)|^{p} x^{\alpha p} d x,
$$

with a constant $C$ independent of $f$.

It follows from the theorem that $\mathcal{T}_{\mu}^{\nu}=T_{\mu, \nu}$ on $L^{2}(0, \infty)$, and so we also call $T_{\mu, \nu}$ the transplantation operator for the Hankel transform from $\nu$ to $\mu$. 
Our first theorem is stated in terms of $T_{\mu, \nu}$ and will be proved in the next section.

THEOREM 1. Let $f$ be a function on $(0, \infty)$ of bounded variation on every interval $[a, b] \subset(0, \infty)$. Put

$$
A=\int_{0}^{\infty}|f(x)| d x, \quad B=\sup _{0<a<b<\infty} \int_{(a, b]} x|d f(x)|,
$$

and suppose that $A, B<\infty$, where $\int_{(a, b]} x|d f(x)|$ is the Lebesgue-Stieltjes integral with respect to the total variation $|d f|$ of the Lebesgue-Stieltjes measure df generated by $f$ modified so as to be right continuous.

(i) If $\mu \geq-1 / 2$ and $\nu>-1 / 2$, then $T_{\mu, \nu} f \in L^{1}(0, \infty)$ and

$$
\int_{0}^{\infty}\left|T_{\mu, \nu} f(x)\right| d x \leq C(A+B)
$$

with a positive constant $C$ depending only on $\mu$ and $\nu$.

(ii) If $\mu \geq-1 / 2$, then

$$
T_{\mu,-1 / 2} f(x)=\frac{K_{\mu,-1 / 2}}{x} \int_{0}^{x} f(t) d t+h(x)
$$

for a.e. $x>0$ with a function $h \in L^{1}(0, \infty)$ satisfying (6) with $h$ instead of $T_{\mu, \nu} f$.

There are transplantation theorems for other orthogonal expansions, e.g., Askey and Wainger [2], Askey [1], Gilbert [7], Muckenhoupt [22], Kanjin [12] and Miyachi [19], [20]. The author [14] quite recently proved the transplantation theorem for the Hankel transform on the real Hardy space, which will be used in our consideration of the Cesàro operators $\mathcal{C}_{\mu}^{\nu}$. Let us recall it here.

Let $H^{1}\left(\mathbb{R}_{+}^{2}\right)$ be the Hardy space on the upper half plane $\mathbb{R}_{+}^{2}=\{z=$ $x+i t ; t>0\}$, that is, the space of analytic functions $F(z)$ on $\mathbb{R}_{+}^{2}$ such that $\|F\|_{H^{1}\left(\mathbb{R}_{+}^{2}\right)}=\sup _{t>0} \int_{-\infty}^{\infty}|F(x+i t)| d x<\infty$. The real Hardy space $H^{1}(\mathbb{R})$ is the space of the boundary functions $f(x)=\Re F(x)$ of the real parts $\Re F(z)$ of $F \in H^{1}\left(\mathbb{R}_{+}^{2}\right)$ with the norm $\|f\|_{H^{1}(\mathbb{R})}=\|F\|_{H^{1}\left(\mathbb{R}_{+}^{2}\right)}$. Let $H^{1}(0, \infty)$ be the space defined by

$$
H^{1}(0, \infty)=\left\{\left.h\right|_{(0, \infty)} ; h \in H^{1}(\mathbb{R}), \operatorname{supp} h \subset[0, \infty)\right\},
$$

where $[0, \infty)$ is the closed half line, and we endow the space with the norm $\|f\|_{H^{1}(0, \infty)}=\|h\|_{H^{1}(\mathbb{R})}$, where $h \in H^{1}(\mathbb{R}), \operatorname{supp} h \subset[0, \infty)$ and $f=\left.h\right|_{(0, \infty)}$. We remark that $H^{1}(0, \infty)=\left\{\left.h\right|_{(0, \infty)} ; h \in H^{1}(\mathbb{R})\right.$, even $\}$ and $c_{1}\|h\|_{H^{1}(\mathbb{R})} \leq$ $\|f\|_{H^{1}(0, \infty)} \leq c_{2}\|h\|_{H^{1}(\mathbb{R})}$ with positive constants $c_{1}$ and $c_{2}$, where $f=\left.h\right|_{(0, \infty)}$ and $h \in H^{1}(\mathbb{R})$ is even. For this fact, see [4, Chapter III, Lemma 7.40]. 
[C] (Kanjin [14]).

(i) Let $\mu \geq-1 / 2$ and $\nu>-1 / 2$. Then $\mathcal{T}_{\mu}^{\nu}$, initially defined on $H^{1}(0, \infty)$ $\cap L^{2}(0, \infty)$, uniquely extends to a bounded operator on $H^{1}(0, \infty)$, still denoted by $\mathcal{T}_{\mu}^{\nu}$, so that

$$
\left\|\mathcal{T}_{\mu}^{\nu} f\right\|_{H^{1}(0, \infty)} \leq C\|f\|_{H^{1}(0, \infty)}
$$

for $f \in H^{1}(0, \infty)$ with a constant $C$ depending only on $\mu$ and $\nu$.

(ii) If $\mu \geq-1 / 2$, then $\mathcal{T}_{\mu}^{-1 / 2}$ uniquely extends to a bounded operator from $H^{1}(0, \infty)$ to $L^{1}(0, \infty)$, that is,

$$
\left\|\mathcal{T}_{\mu}^{-1 / 2} f\right\|_{L^{1}(0, \infty)} \leq C\|f\|_{H^{1}(0, \infty)}
$$

for $f \in H^{1}(0, \infty)$ with a constant $C$ depending only on $\mu$ and $\nu$.

We now turn to our Cesàro operators $\mathcal{C}_{\mu}^{\nu}$. Let $\mathcal{F} g$ be the Fourier transform of $g: \mathcal{F} g(\xi)=(1 / \sqrt{2 \pi}) \int_{-\infty}^{\infty} g(t) e^{-i t \xi} d t$, and $\mathcal{F}^{-1} g$ be the inverse Fourier transform: $\mathcal{F}^{-1} g(\xi)=(1 / \sqrt{2 \pi}) \int_{-\infty}^{\infty} g(t) e^{i t \xi} d t$. The classical Cesàro operator $\mathcal{C}$ is defined as follows:

$$
\mathcal{F}(\mathcal{C} g)(y)=\frac{1}{y} \int_{0}^{y} \mathcal{F} g(\xi) d \xi, \quad-\infty<y<\infty
$$

One of the results on the operator $\mathcal{C}$ is the following:

[D] (Giang and Móricz [6]).

(i) The Cesàro operator $\mathcal{C}$ satisfies $\mathcal{C} g(x)=S g(x)$ and $\mathcal{C} g(-x)=$ $S(g(-\cdot))(x)$ for a.e. $x>0$ and for all $g \in L^{2}(-\infty, \infty)$. In particular, $\mathcal{C}$ uniquely extends to a bounded operator on $L^{p}(-\infty, \infty), 1 \leq p<\infty$.

(ii) $\mathcal{C}$ uniquely extends to a bounded operator on $H^{1}(\mathbb{R})$, and so does $S$.

It follows that $\mathcal{F} g_{\mathrm{e}}$ and $\mathcal{F}^{-1} g_{\mathrm{e}}$ are even, and $\mathcal{F} g_{\mathrm{o}}$ and $\mathcal{F}^{-1} g_{\mathrm{o}}$ are odd. Further, we see that for $g \in L^{2}(\mathbb{R}), \mathcal{F} g_{\mathrm{e}}(y)=\mathcal{H}_{-1 / 2}\left(R g_{\mathrm{e}}\right)(y), \mathcal{F}^{-1} g_{\mathrm{e}}(y)=$ $\mathcal{H}_{-1 / 2}\left(R g_{\mathrm{e}}\right)(y), \mathcal{F}_{g_{\mathrm{o}}}(y)=\mathcal{H}_{1 / 2}\left(R g_{\mathrm{o}}\right)(y)$, and $\mathcal{F}^{-1} g_{\mathrm{o}}(y)=-\mathcal{H}_{1 / 2}\left(R g_{\mathrm{o}}\right)(y)$ for a.e. $y>0$. The function $(1 / x) \int_{0}^{x} g(y) d y$ of $x$ is even or odd for $g$ even or odd, respectively. It follows from these facts that for $g \in L^{2}(\mathbb{R})$,

$$
\mathcal{C} g(x)= \begin{cases}\mathcal{C}_{-1 / 2}^{-1 / 2}\left(R g_{\mathrm{e}}\right)(x)+\mathcal{C}_{1 / 2}^{1 / 2}\left(R g_{\mathrm{o}}\right)(x) & (\text { a.e. } x>0), \\ \mathcal{C}_{-1 / 2}^{-1 / 2}\left(R g_{\mathrm{e}}\right)(-x)-\mathcal{C}_{1 / 2}^{1 / 2}\left(R g_{\mathrm{o}}\right)(-x) & (\text { a.e. } x<0),\end{cases}
$$

and in particular $\mathcal{C} g_{\mathrm{e}}(x)=\mathcal{C}_{-1 / 2}^{-1 / 2}\left(R g_{\mathrm{e}}\right)(x)$ and $\mathcal{C} g_{\mathrm{o}}(x)=\mathcal{C}_{1 / 2}^{1 / 2}\left(R g_{\mathrm{o}}\right)(x)$ for a.e. $x>0$. Thus, the Cesàro operators $\mathcal{C}_{\mu}^{\nu}$ for the Hankel transform are generalizations of the classical Cesàro operator $\mathcal{C}$, and the two operators $\mathcal{C}_{-1 / 2}^{-1 / 2}$ and $\mathcal{C}_{1 / 2}^{1 / 2}$ which are equal to $S$ are bounded on $L^{p}(0, \infty), 1 \leq p<\infty$, and on $H^{1}(0, \infty)$ by [D]. This inspires us to investigate the boundedness of $\mathcal{C}_{\mu}^{\nu}$. 
By Theorem 1, the following simple lemma combining the Cesàro operators with the transplantation operators allows us to get our next theorem; their proofs will be given in the next section.

Lemma. Let $\mu, \nu \geq-1 / 2$. Then $\mathcal{C}_{\mu}^{\nu}=\mathcal{T}_{\mu}^{\nu} S$ on $L^{2}(0, \infty)$, where $S$ is defined in (1).

\section{THEOREM 2.}

(i) Let $\mu \geq-1 / 2$ and $\nu>-1 / 2$. If $1 \leq p<\infty$, then $\mathcal{C}_{\mu}^{\nu}$, initially defined on $L^{p}(0, \infty) \cap L^{2}(0, \infty)$, uniquely extends to a bounded operator on $L^{p}(0, \infty)$, still denoted by $\mathcal{C}_{\mu}^{\nu}$, so that

$$
\left\|\mathcal{C}_{\mu}^{\nu} f\right\|_{L^{p}(0, \infty)} \leq C\|f\|_{L^{p}(0, \infty)}
$$

for $f \in L^{p}(0, \infty)$ with a constant $C$ depending only on $\mu, \nu$ and $p$. Further, $\mathcal{C}_{\mu}^{\nu}$, initially defined on $H^{1}(0, \infty) \cap L^{2}(0, \infty)$, uniquely extends to a bounded operator on $H^{1}(0, \infty)$, that is,

$$
\left\|\mathcal{C}_{\mu}^{\nu} f\right\|_{H^{1}(0, \infty)} \leq C\|f\|_{H^{1}(0, \infty)}
$$

for $f \in H^{1}(0, \infty)$ with a constant $C$ depending only on $\mu$ and $\nu$.

(ii) Let $\mu \geq-1 / 2$. If $1<p<\infty$, then $\mathcal{C}_{\mu}^{-1 / 2}$ uniquely extends to a bounded operator on $L^{p}(0, \infty)$, that is,

$$
\left\|\mathcal{C}_{\mu}^{-1 / 2} f\right\|_{L^{p}(0, \infty)} \leq C\|f\|_{L^{p}(0, \infty)}
$$

for $f \in L^{p}(0, \infty)$ with a constant $C$ depending only on $\mu$ and $p$. In the case $p=1$, it follows that $\mathcal{C}_{\mu}^{-1 / 2}$ uniquely extends to a bounded operator from $H^{1}(0, \infty)$ to $L^{1}(0, \infty)$, that is,

$$
\left\|\mathcal{C}_{\mu}^{-1 / 2} f\right\|_{L^{1}(0, \infty)} \leq C\|f\|_{H^{1}(0, \infty)}
$$

for $f \in H^{1}(0, \infty)$ with a constant $C$ depending only on $\mu$.

For related topics, see Goldberg [8], Georgakis [5], Móricz [21], Liflyand and Móricz [17], and Kanjin [13].

Another motivation for studying the Cesàro operators $\mathcal{C}_{\mu}^{\nu}$ is the series of results on the periodic case: Hardy [10] proved that if $\sum_{n=0}^{\infty} a_{n} \cos n x$ is the Fourier series of a function in $L^{p}(0, \pi)$, then so is $\sum_{n=0}^{\infty}(T a)_{n} \cos n x$ for $1 \leq p<\infty$, where $(T a)_{0}=a_{0},(T a)_{n}=\left(a_{1}+\cdots+a_{n}\right) / n, n=$ $1,2, \ldots$, and the same is true for sine series. These may correspond to the boundedness of $\mathcal{C}_{-1 / 2}^{-1 / 2}$ and $\mathcal{C}_{1 / 2}^{1 / 2}$. Kinukawa and Igari [15] showed that if $\sum_{n=1}^{\infty} b_{n} \sin n x$ is a Fourier series, then $\sum_{n=1}^{\infty}(T b)_{n} \cos n x$ is a Fourier series. This case may correspond to the boundedness of $\mathcal{C}_{-1 / 2}^{1 / 2}$. Loo [18] remarked that $\sum_{n=1}^{\infty}(T a)_{n} \sin n x$ is not necessarily a Fourier series even if $\sum_{n=1}^{\infty} a_{n} \cos n x$ is one, which may be the reason why we discuss the cases 
$\nu>-1 / 2$ and $\nu=-1 / 2$ separately. Siskakis [24] obtained the same type of theorem in the Hardy space $H^{1}$ of the unit disc.

2. Proofs. We shall first give a proof of Theorem 1, and then of the Lemma. Lastly, we shall prove Theorem 2 by using Theorem 1 and the Lemma.

Proof of Theorem 1. Let $\mu, \nu \geq-1 / 2$. We may suppose $\mu \neq \nu$. It is enough to consider the part

$$
\lim _{\delta \rightarrow+0} \int_{\delta<|x-y|} f(y) \widetilde{I}_{\mu, \nu}(x, y) d y
$$

of the operator $T_{\mu, \nu}$ of (4). Suppose that

$$
A=\int_{0}^{\infty}|f(x)| d x<\infty, \quad B=\sup _{0<a<b<\infty} \int_{(a, b]} x|d f(x)|<\infty .
$$

Let $x>0$ and let $\delta$ be an arbitrarily small positive number such that $0<$ $\delta<x / 2$. We divide the integral into three parts:

$$
\begin{aligned}
\int_{\delta<|x-y|} f(y) \widetilde{I}_{\mu, \nu}(x, y) d y & =\left\{\int_{0}^{x / 2}+\int_{\delta<|x-y|<x / 2}+\int_{3 x / 2}^{\infty}\right\} f(y) \widetilde{I}_{\mu, \nu}(x, y) d y \\
& =L(x)+I_{\delta}(x)+U(x), \text { say. }
\end{aligned}
$$

We first estimate

$$
L(x)=2^{-1} K_{\mu, \nu} \int_{0}^{x / 2}\left(\frac{y}{x}\right)^{\nu+1 / 2}\left(\frac{1}{x-y}+\frac{1}{x+y}\right) F_{\mu, \nu}(x, y) f(y) d y
$$

where we put

$$
F_{\mu, \nu}(x, y)=F\left(\frac{\nu-\mu}{2}, \frac{\mu+\nu}{2} ; \nu+1 ; \frac{y^{2}}{x^{2}}\right)
$$

for simplicity. If $0<y<x / 2$, then $1 /(x-y) \leq 2 / x, 1 /(x+y) \leq 1 / x$ and $\left|F_{\mu, \nu}(x, y)\right| \leq C$. This leads to

$$
|L(x)| \leq \frac{C}{x} \int_{0}^{x / 2}\left(\frac{y}{x}\right)^{\nu+1 / 2}|f(y)| d y .
$$

Here and below, $C$ denotes a positive constant depending only on $\mu$ and $\nu$ which may be different at different occurrences, even in the same chain of inequalities.

We remark that

$$
\int_{0}^{\infty}\left|\frac{1}{x} \int_{0}^{x}\left(\frac{y}{x}\right)^{\alpha} f(y) d y\right| d x \leq \frac{1}{\alpha} \int_{0}^{\infty}|f(x)| d x
$$


where $\alpha>0$. For, by inverting the order of integration, we see that the integral on the left-hand side is bounded by

$$
\int_{0}^{\infty} \int_{y}^{\infty} x^{-(\alpha+1)} d x|f(y)| y^{\alpha} d y
$$

which is $(1 / \alpha) \int_{0}^{\infty}|f(y)| d y$ if $\alpha>0$. By (8), $L(x)$ is integrable and

$$
\int_{0}^{\infty}|L(x)| d x \leq C A
$$

when $\mu \geq-1 / 2$ and $\nu>-1 / 2$.

Let us discuss the case $\nu=-1 / 2$ for $L(x)$. We write

$$
\begin{aligned}
L(x)= & K_{\mu} \frac{2}{x} \int_{0}^{x / 2} F_{\mu}(x, y) f(y) d y+K_{\mu} \int_{0}^{x / 2}\left(\frac{1}{x-y}-\frac{1}{x}\right) F_{\mu}(x, y) f(y) d y \\
& +K_{\mu} \int_{0}^{x / 2}\left(\frac{1}{x+y}-\frac{1}{x}\right) F_{\mu}(x, y) f(y) d y \\
= & L_{1}(x)+L_{2}(x)+L_{3}(x), \quad \text { say, }
\end{aligned}
$$

where $K_{\mu}=K_{\mu,-1 / 2} / 2$ and $F_{\mu}(x, y)=F_{\mu,-1 / 2}(x, y)$. Since $|1 /(x-y)-1 / x| \leq$ $2 y / x^{2},|1 /(x+y)-1 / x| \leq y / x^{2}$ and $\left|F_{\mu}(x, y)\right| \leq C$ for $0<y<x / 2$, it follows that

$$
\left|L_{2}(x)\right|+\left|L_{3}(x)\right| \leq \frac{C}{x} \int_{0}^{x / 2} \frac{y}{x}|f(y)| d y .
$$

By (8) with $\alpha=1$, we see that $L_{2}(x)$ and $L_{3}(x)$ are integrable, and that $\int_{0}^{\infty}\left|L_{j}(x)\right| d x \leq C A, j=2,3$. We decompose $L_{1}(x)$ as follows:

$$
\begin{aligned}
L_{1}(x) & =\frac{2 K_{\mu}}{x} \int_{0}^{x} f(y) d y+\frac{-2 K_{\mu}}{x} \int_{x / 2}^{x} f(y) d y+K_{\mu} \frac{2}{x} \int_{0}^{x / 2}\left(F_{\mu}(x, y)-1\right) f(y) d y \\
& =\frac{2 K_{\mu}}{x} \int_{0}^{x} f(y) d y+L_{11}(x)+L_{12}(x), \quad \text { say. }
\end{aligned}
$$

It follows from $\left|F_{\mu}(x, y)-1\right| \leq C(y / x)^{2}$ that $\int_{0}^{\infty}\left|L_{12}(x)\right| d x \leq C A$ by (8) with $\alpha=2$. The estimate $\left|L_{11}(x)\right| \leq C \int_{x / 2}^{\infty}|f(y)| d y / y$ implies $\int_{0}^{\infty}\left|L_{11}(x)\right| d x \leq$ $C A$. Therefore,

$$
L(x)=\frac{2 K_{\mu}}{x} \int_{0}^{x} f(y) d y+h(x)
$$

with some integrable function $h$ satisfying $\int_{0}^{\infty}|h(x)| d x \leq C A$ when $\nu=-1 / 2$ and $\mu \geq-1 / 2$. 
We next estimate

$$
U(x)=2^{-1} K_{\nu, \mu} \int_{3 x / 2}^{\infty}\left(\frac{x}{y}\right)^{\mu+1 / 2}\left(\frac{1}{y-x}+\frac{1}{y+x}\right) F_{\nu, \mu}(y, x) f(y) d y
$$

of (7) for $\mu \geq-1 / 2$ and $\nu \geq-1 / 2$. It follows that $1 /(y-x) \leq 3 / y$, $1 /(x+y) \leq 1 / y$ and $\left|F_{\nu, \mu}(y, x)\right| \leq C$ for $3 x / 2<y$, which implies $|U(x)| \leq$ $C \int_{3 x / 2}^{\infty}|f(y)| d y / y$. This leads to integrability of $U(x)$ and

$$
\int_{0}^{\infty}|U(x)| d x \leq C A
$$

for $\mu \geq-1 / 2$ and $\nu \geq-1 / 2$.

We now turn to estimating $I_{\delta}(x)$ of $(7)$. We put

$$
\widetilde{F}_{\mu, \nu}(x, y)=2^{-1} K_{\mu, \nu}(y / x)^{\nu+1 / 2} F_{\mu, \nu}(x, y)
$$

for $0<y<x$, and $\widetilde{F}_{\mu, \nu}(x, y)=\widetilde{F}_{\nu, \mu}(y, x)$ for $0<x<y$, and then by the definition of the kernel $\widetilde{I}_{\mu, \nu}(x, y)$ we have

$$
\begin{aligned}
I_{\delta}(x)= & \int_{x / 2}^{x-\delta} \frac{\widetilde{F}_{\mu, \nu}(x, y)}{x-y} f(y) d y+\int_{x+\delta}^{3 x / 2} \frac{\widetilde{F}_{\mu, \nu}(x, y)}{y-x} f(y) d y \\
& +\int_{x / 2}^{x-\delta} \frac{\widetilde{F}_{\mu, \nu}(x, y)}{x+y} f(y) d y+\int_{x+\delta}^{3 x / 2} \frac{\widetilde{F}_{\mu, \nu}(x, y)}{y+x} f(y) d y \\
= & I_{\delta}^{1}(x)+I_{\delta}^{2}(x)+I_{\delta}^{3}(x)+I_{\delta}^{4}(x), \quad \text { say. }
\end{aligned}
$$

We first deal with $I_{\delta}^{3}(x)$ and $I_{\delta}^{4}(x)$. Since

$$
\lim _{z \rightarrow 1-} F(\alpha, \beta ; \gamma ; z)=\frac{\Gamma(\gamma) \Gamma(\gamma-\alpha-\beta)}{\Gamma(\gamma-\alpha) \Gamma(\gamma-\beta)}
$$

for $\Re(\gamma-\alpha-\beta)>0[16,(9.3 .4)]$, it follows from $\nu+1-(\nu-\mu) / 2-(\nu+\mu) / 2=1$ and $\mu+1-(\mu-\nu) / 2-(\mu+\nu) / 2=1$ that

$$
\begin{aligned}
& \left|F\left(\frac{\nu-\mu}{2}, \frac{\nu+\mu}{2} ; \nu+1 ; \frac{y^{2}}{x^{2}}\right)\right| \leq C, \quad 0<y<x, \\
& \left|F\left(\frac{\mu-\nu}{2}, \frac{\mu+\nu}{2} ; \mu+1 ; \frac{x^{2}}{y^{2}}\right)\right| \leq C, \quad 0<x<y .
\end{aligned}
$$

We have $\left|\widetilde{F}_{\mu, \nu}(x, y)\right| \leq C$ for $x, y>0$, which implies that the integrands in $I_{\delta}^{3}(x)$ and $I_{\delta}^{4}(x)$ are bounded by $C f(y) / y$. Therefore, the following limits exist for every $x>0$ : 


$$
\begin{aligned}
& \lim _{\delta \rightarrow+0} I_{\delta}^{3}(x)=\int_{x / 2}^{x} \frac{\widetilde{F}_{\mu, \nu}(x, y)}{x+y} f(y) d y=I^{3}(x), \quad \text { say, } \\
& \lim _{\delta \rightarrow+0} I_{\delta}^{4}(x)=\int_{x}^{3 x / 2} \frac{\widetilde{F}_{\mu, \nu}(x, y)}{y+x} f(y) d y=I^{4}(x), \quad \text { say, }
\end{aligned}
$$

and $\left|I^{3}(x)\right| \leq C \int_{x / 2}^{\infty}|f(y)| d y / y$ and $\left|I^{4}(x)\right| \leq C \int_{x}^{\infty}|f(y)| d y / y$, which shows that $I^{3}(x)$ and $I^{4}(x)$ are integrable functions and

$$
\int_{0}^{\infty}\left(\left|I^{3}(x)\right|+\left|I^{4}(x)\right|\right) d x \leq C A
$$

for $\mu \geq-1 / 2$ and $\nu \geq-1 / 2$.

We turn to estimating the sum $I_{\delta}^{1}(x)+I_{\delta}^{2}(x)$ in (12). We put $K_{\mu, \nu}^{\prime}=$ $2[(\nu-\mu) \Gamma((\mu-\nu) / 2) \Gamma((\nu-\mu) / 2)]^{-1}$ for $\mu \geq-1 / 2$ and $\nu \geq-1 / 2$. Then, noting $K_{\mu, \nu}^{\prime}=-K_{\nu, \mu}^{\prime}$, we have

$$
I_{\delta}^{1}(x)+I_{\delta}^{2}(x)=K_{\mu, \nu}^{\prime} \int_{\delta<|x-y|<x / 2} \frac{f(y)}{x-y} d y+I_{\delta}^{11}(x)+I_{\delta}^{12}(x)+I_{\delta}^{21}(x)+I_{\delta}^{22}(x),
$$

where

$$
\begin{aligned}
& I_{\delta}^{11}(x)=2^{-1} K_{\mu, \nu} \int_{x / 2}^{x-\delta}\left(\left(\frac{y}{x}\right)^{\nu+1 / 2}-1\right) \frac{F_{\mu, \nu}(x, y)}{x-y} f(y) d y \\
& I_{\delta}^{21}(x)=2^{-1} K_{\nu, \mu} \int_{x+\delta}^{3 x / 2}\left(\left(\frac{x}{y}\right)^{\mu+1 / 2}-1\right) \frac{F_{\nu, \mu}(x, y)}{y-x} f(y) d y \\
& I_{\delta}^{12}(x)=\int_{x / 2}^{x-\delta}\left(2^{-1} K_{\mu, \nu} F_{\mu, \nu}(x, y)-K_{\mu, \nu}^{\prime}\right) \frac{f(y)}{x-y} d y \\
& I_{\delta}^{22}(x)=\int_{x+\delta}^{3 x / 2}\left(2^{-1} K_{\nu, \mu} F_{\nu, \mu}(x, y)-K_{\nu, \mu}^{\prime}\right) \frac{f(y)}{y-x} d y .
\end{aligned}
$$

We shall show that the limit function

$$
I^{i j}(x)=\lim _{\delta \rightarrow+0} I_{\delta}^{i j}(x)
$$

exists and $\int_{0}^{\infty}\left|I^{i j}(x)\right| d x \leq C A$ for $i, j=1,2$. Let us estimate $I_{\delta}^{11}(x)$. The integrand in $I_{\delta}^{11}(x)$ is estimated as follows:

$$
\left|\left(\left(\frac{y}{x}\right)^{\nu+1 / 2}-1\right) \frac{F_{\mu, \nu}(x, y)}{x-y} f(y)\right| \leq C \frac{|f(y)|}{x}
$$

for $0<y<x$ when $\mu \geq-1 / 2$ and $\nu \geq-1 / 2$. Here, we used the fact $\left|F_{\mu, \nu}(x, y)\right| \leq C$ for $0<y<x$ and the simple inequalities $1-u^{\alpha} \leq \alpha(1-u)$ 
for $0<u<1$ when $\alpha \geq 1$, and $1-u^{\alpha} \leq 1-u$ for $0<u<1$ when $0<\alpha<1$. Thus the limit $I^{11}(x)$ exists for every $x>0$, and

$$
\left|I^{11}(x)\right| \leq C \int_{x / 2}^{x} \frac{|f(y)|}{x} d y \leq C \int_{x / 2}^{\infty} \frac{|f(y)|}{y} d y
$$

which leads to the integrability of $I^{11}(x)$ and $\int_{0}^{\infty}\left|I^{11}(x)\right| d x \leq C A$ for $\mu \geq$ $-1 / 2$ and $\nu \geq-1 / 2$.

For $I_{\delta}^{12}(x)$, we use the following asymptotic formula [16, (9.7.5)]:

$$
\begin{aligned}
& F(\alpha, \beta ; \alpha+\beta+1 ; z)=\frac{\Gamma(\alpha+\beta+1)}{\Gamma(\alpha+1) \Gamma(\beta+1)} \\
& \quad+\frac{-\Gamma(\alpha+\beta+1)}{\Gamma(\alpha) \Gamma(\beta)} \sum_{k=0}^{\infty} \frac{(\alpha+1)_{k}(\beta+1)_{k}}{(k+1) ! k !}[\psi(k+1)+\psi(k+2) \\
& \quad-\psi(\alpha+k+1)-\psi(\beta+k+1)-\log (1-z)](1-z)^{k+1}, \\
& |z-1|<1,|\arg (1-z)|<\pi, n=0,1,2, \ldots, \alpha, \beta \neq 0,-1,-2, \ldots,
\end{aligned}
$$

where $\psi(z)=\Gamma^{\prime}(z) / \Gamma(z)$ is the logarithmic derivative of the gamma function. It is known that $\psi(n+1)=-\gamma+\sum_{k=1}^{n} 1 / k$ for $n=1,2, \ldots$, where $\gamma$ is Euler's constant. We apply this formula to the term $2^{-1} K_{\mu, \nu} F_{\mu, \nu}(x, y)$ in $I_{\delta}^{12}(x)$. Since $\alpha=(\nu-\mu) / 2, \beta=(\nu+\mu) / 2$ and $z=y^{2} / x^{2}$ in our case, we see $K_{\mu, \nu}^{\prime}$ is equal to the constant term of the asymptotic expansion of $2^{-1} K_{\mu, \nu} F_{\mu, \nu}(x, y)$, and

$$
\begin{aligned}
\left|2^{-1} K_{\mu, \nu} F_{\mu, \nu}(x, y)-K_{\mu, \nu}^{\prime}\right| & \leq C\left|1-\frac{y^{2}}{x^{2}}\right|\left|\log \left(1-\frac{y^{2}}{x^{2}}\right)\right| \\
& \leq C \frac{|x-y|}{x}\left|\log \left(1-\frac{y}{x}\right)\right|
\end{aligned}
$$

for $0<y<x$. Here, the following conditions must be satisfied:

$$
\alpha=(\nu-\mu) / 2 \neq 0,-1,-2, \ldots, \quad \beta=(\nu+\mu) / 2 \neq 0,-1,-2, \ldots
$$

Because of $\mu, \nu \geq-1 / 2$ and $\mu \neq \nu$, the conditions above are equivalent to $\nu-\mu \neq-2,-4, \ldots$ and $\nu+\mu \neq 0$. But if $\nu+\mu=0$, then by the definition of the hypergeometric functions $F_{\mu, \nu}(x, y)$ is constantly 1 , and $2^{-1} K_{\mu, \nu}=K_{\mu, \nu}^{\prime}$. Thus, (15) holds trivially and so the integrand in $I_{\delta}^{12}(x)$ is bounded by $C|\log (1-y / x)||f(y)| / x$ when $\mu, \nu \geq-1 / 2$ and $\nu-\mu \neq-2,-4, \ldots$ Consider the integral

$$
\int_{0}^{\infty} \frac{1}{x} \int_{0}^{x}\left|\log \left(1-\frac{y}{x}\right)\right||f(y)| d y d x
$$


which is equal to

$$
\begin{aligned}
\int_{0}^{\infty} \int_{y}^{\infty} \frac{1}{x}\left|\log \left(1-\frac{y}{x}\right)\right| d x|f(y)| d y & =\int_{0}^{1}|\log (1-t)| t d t \int_{0}^{\infty}|f(y)| d y \\
& \leq C \int_{0}^{\infty}|f(y)| d y
\end{aligned}
$$

This shows that the function $|\log (1-y / x)||f(y)| / x$ of $y$ is integrable for a.e. $x>0$, and thus the limit $I^{12}(x)$ exists for a.e. $x>0$ and $\int_{0}^{\infty}\left|I^{12}(x)\right| d x \leq C A$ where $\nu-\mu \neq-2,-4, \ldots$. Applying the same argument to $I_{\delta}^{21}(x)$ and $I_{\delta}^{22}(x)$, we find that the limit functions $I^{2 j}(x), j=1,2$, are integrable and $\int_{0}^{\infty}\left|I^{2 j}(x)\right| d x \leq C A$ for $j=1,2$ when $\mu-\nu \neq-2,-4, \ldots$ Therefore,

$$
\lim _{\delta \rightarrow+0}\left(I_{\delta}^{1}(x)+I_{\delta}^{2}(x)\right)=K_{\mu, \nu}^{\prime} \lim _{\delta \rightarrow+0} \int_{\delta<|x-y|<x / 2} \frac{f(y)}{x-y} d y+h(x),
$$

with some integrable function $h(x)$ satisfying $\int_{0}^{\infty}|h(x)| d x \leq C A$ provided $\mu-\nu \neq \pm 2, \pm 4, \ldots$.

The condition $B=\sup _{0<a<b<\infty} \int_{(a, b]} x|d f(x)|<\infty$ implies the integrability of the part $\lim _{\delta \rightarrow+0} \int_{\delta<|x-y|<x / 2} f(y) /(x-y) d y$ in (16). The proof is in $[11$, p. 2 , lines 18-23], but for the reader's convenience we give it here. We have

$$
\int_{\delta<|x-y|<x / 2} \frac{f(y)}{x-y} d y=\int_{\delta}^{x / 2} \frac{f(x-u)-f(x+u)}{u} d u=-\int_{\delta}^{x / 2} \int_{(x-u, x+u]} d f(w) \frac{d u}{u},
$$

which implies

$$
\begin{aligned}
\int_{\delta<|x-y|<x / 2} \frac{f(y)}{x-y} d y \mid & \leq \int_{\delta}^{x / 2} \int_{(x-u, x+u]}|d f(w)| \frac{d u}{u} \\
& \leq \int_{0}^{x / 2} \int_{(x-u, x+u]}|d f(w)| \frac{d u}{u}=g(x), \quad \text { say. }
\end{aligned}
$$

The function $g(x)$ is integrable. For, we have

$$
\begin{aligned}
g(x) & =\int_{(x / 2, x]} \int_{x-w}^{x / 2} \frac{d u}{u}|d f(w)|+\int_{(x, 3 x / 2]} \int_{w-x}^{x / 2} \frac{d u}{u}|d f(w)| \\
& =\int_{(x / 2,3 x / 2]} \log \frac{x}{2|w-x|}|d f(w)|,
\end{aligned}
$$

and thus for $0<a<b<\infty$ such that $a$ is small enough and $b$ is large 
enough, we have

$$
\begin{aligned}
\int_{a}^{b} g(x) d x & =\int_{a}^{b} \int_{(x / 2,3 x / 2]} \log \frac{x}{2|w-x|}|d f(w)| \\
& =\left\{\int_{(a / 2,3 a / 2]} \int_{a}^{2 w}+\int_{(3 a / 2, b / 2]} \int_{2 w / 3}^{2 w}+\int_{(b / 2,3 b / 2]} \int_{2 w / 3}^{b}\right\} \log \frac{x}{2|w-x|} d x|d f(w)| \\
& \leq \int_{2 / 3}^{2} \log \frac{t}{2|1-t|} d t \int_{(a / 2,3 b / 2]} w|d f(w)|
\end{aligned}
$$

By the assumption $\int_{(a / 2,3 b / 2]} w|d f(w)| \leq B$ and $\int_{2 / 3}^{2} \log (t /(2|1-t|)) d t<\infty$, we have $\int_{0}^{\infty} g(x) d x \leq C B$. Thus, $\lim _{\delta \rightarrow+0} \int_{\delta<|x-y|<x / 2} f(y) /(x-y) d y$ exists for a.e. $x>0$, and the limit function, say $f_{1}(x)$, satisfies $\int_{0}^{\infty}\left|f_{1}(x)\right| d x \leq C B$. This together with (13) and (16) implies that $I(x)=\lim _{\delta \rightarrow+0} I_{\delta}(x)$ exists for a.e. $x>0$ and

$$
\int_{0}^{\infty}|I(x)| d x \leq C(A+B)
$$

if $\mu, \nu \geq-1 / 2$ and $\nu-\mu \neq \pm 2, \pm 4, \ldots$.

Let us summarize the results (9), (10), (11) and (17) that we have already obtained: Suppose that

$$
A=\int_{0}^{\infty}|f(x)| d x<\infty, \quad B=\sup _{0<a<b<\infty} \int_{(a, b]} x|d f(x)|<\infty .
$$

If $\mu-\nu \neq \pm 2, \pm 4, \ldots$, then

$$
\begin{aligned}
\lim _{\delta \rightarrow+0} & \int_{\delta<|x-y|} f(y) \widetilde{I}_{\mu, \nu}(x, y) d y \\
& = \begin{cases}h_{1}(x) & (\mu \geq-1 / 2, \nu>-1 / 2), \\
\frac{K_{\mu,-1 / 2}}{x} \int_{0}^{x} f(y) d y+h_{2}(x) & (\mu \geq-1 / 2, \nu=-1 / 2),\end{cases}
\end{aligned}
$$

for a.e. $x>0$, where $h_{1}(x)$ is an integrable function satisfying (17) with $h_{1}$ instead of $I$, and similarly for $h_{2}$.

We continue the proof of Theorem 1. Let us deal with the case $\mu-\nu$ $= \pm 2 k$ for some positive integer $k$. Although the proof of this case is essentially included in [23, Theorems 3 and 4$]$, we give it briefly. The representation (5) shows that for $\mu=\nu+2 k, k=1,2, \ldots$, 


$$
\widetilde{I}_{\nu+2 k, \nu}(x, y)= \begin{cases}\frac{1}{x} \sum_{j=0}^{k-1} \gamma_{j}\left(\frac{y}{x}\right)^{2 j+\nu+1 / 2} & (0<y<x), \\ 0 & (0<x<y),\end{cases}
$$

where $\gamma_{j}$ are constants and $\gamma_{0}=2 \Gamma(\nu+k+1) /\{\Gamma(\nu+1) \Gamma(k)\}$, and for $\mu=\nu-2 k, k=1,2, \ldots$,

$$
\widetilde{I}_{\nu-2 k, \nu}(x, y)= \begin{cases}0 & (0<x<y), \\ \frac{1}{y} \sum_{j=0}^{k-1} \gamma_{j}^{\prime}\left(\frac{x}{y}\right)^{2(j-k)+\nu+1 / 2} & (0<y<x),\end{cases}
$$

where $\gamma_{j}^{\prime}$ are constants (see [23, Section 5]). Let $\mu-\nu=-2 k$ with a positive integer $k$. We use the representation (20) to get

$$
\begin{aligned}
\lim _{\delta \rightarrow+0} \int_{\delta<|x-y|} f(y) \widetilde{I}_{\nu-2 k, \nu}(x, y) d y & =\sum_{j=0}^{k-1} \gamma_{j}^{\prime} \int_{x}^{\infty} \frac{1}{y}\left(\frac{x}{y}\right)^{2(j-k)+\nu+1 / 2} f(y) d y \\
& =h_{3}(x), \text { say, }
\end{aligned}
$$

for every $x>0$. We note that

$$
\int_{0}^{\infty}\left|\int_{x}^{\infty} \frac{1}{y}\left(\frac{x}{y}\right)^{\alpha} f(y) d y\right| d x \leq \frac{1}{1+\alpha} \int_{0}^{\infty}|f(x)| d x
$$

for $\alpha>-1$, which is easily deduced by changing the order of integration. This implies that $\int_{0}^{\infty}\left|h_{3}(x)\right| d x \leq C A$. Suppose that $\mu-\nu=2 k$ with a positive integer $k$. The representation (19) leads to

$$
\begin{aligned}
\lim _{\delta \rightarrow+0} \int_{\delta<|x-y|} f(y) \widetilde{I}_{\nu+2 k, \nu}(x, y) d y & =\sum_{j=0}^{k-1} \frac{\gamma_{j}}{x} \int_{0}^{x}\left(\frac{y}{x}\right)^{2 j+\nu+1 / 2} f(y) d y \\
& =h_{4}(x), \quad \text { say. }
\end{aligned}
$$

If $\nu>-1 / 2$, then $2 j+\nu+1 / 2>0$ for $j=0, \ldots, k-1$, and then the inequality (8) implies that $\int_{0}^{\infty}\left|h_{4}(x)\right| d x \leq C A$. Let us consider the case $\nu=-1 / 2$. Every term with positive $j$ on the right-hand side of (21) is integrable by the same reason above. Thus, when $\mu \geq-1 / 2, \nu=-1 / 2$ and $\mu-\nu=2 k, k=1,2, \ldots$, by noting $\gamma_{0}=K_{\mu,-1 / 2}$ we have

$$
\lim _{\delta \rightarrow+0} \int_{\delta<|x-y|} f(y) \widetilde{I}_{\mu, \nu}(x, y) d y=\frac{K_{\mu,-1 / 2}}{x} \int_{0}^{x} f(y) d y+h_{5}(x),
$$

where $h_{5}$ is some integrable function satisfying $\int_{0}^{\infty}\left|h_{5}(x)\right| d x \leq C A$. Therefore, we see that (18) also holds for the case $\mu-\nu= \pm 2, \pm 4, \ldots$ The proof of Theorem 1 is complete. 
To get the boundedness of $\mathcal{C}_{\mu}^{\nu}$, we prove the Lemma which gives a relation between $\mathcal{T}_{\mu}^{\nu}$ and $\mathcal{C}_{\mu}^{\nu}$.

Proof of Lemma. Let $\mu, \nu \geq-1 / 2$. We first show that $T \mathcal{H}_{\nu} f=\mathcal{H}_{\nu} S$. Let $f \in L^{2}(0, \infty) \cap L^{1}(0, \infty)$. We note that $\mathcal{H}_{\nu} f \in L^{\infty}(0, \infty)$. We have

$$
\begin{aligned}
T \mathcal{H}_{\nu} f(x) & =\frac{1}{x} \int_{0}^{x} \mathcal{H}_{\nu} f(y) d y=\int_{0}^{1} \mathcal{H}_{\nu} f(x u) d u \\
& =\int_{0}^{1} \int_{0}^{\infty} f(s) \sqrt{x u s} J_{\nu}(x u s) d s d u \\
& =\int_{0}^{1} \int_{0}^{\infty} f(t / u) \sqrt{x t} J_{\nu}(x t) d t \frac{d u}{u} .
\end{aligned}
$$

The order of integration in the last iterated integral can be inverted since

$$
\int_{0}^{1} \int_{0}^{\infty}\left|f(t / u) \sqrt{x t} J_{\nu}(x t)\right| d t \frac{d u}{u} \leq C \int_{0}^{1} \int_{0}^{\infty}|f(t / u)| d t \frac{d u}{u}=C \int_{0}^{\infty}|f(y)| d y .
$$

Here, we used the fact that $\left|\sqrt{z} J_{\nu}(z)\right| \leq C$ for $z>0$. Thus, we have

$$
\begin{aligned}
T \mathcal{H}_{\nu} f(x) & =\int_{0}^{\infty} \int_{0}^{1} f(t / u) \frac{d u}{u} \sqrt{x t} J_{\nu}(x t) d t \\
& =\int_{0}^{\infty} \int_{t}^{\infty} f(y) \frac{d y}{y} \sqrt{x t} J_{\nu}(x t) d t=\mathcal{H}_{\nu} S f(x) .
\end{aligned}
$$

The operators $T, S$ and $\mathcal{H}_{\nu}$ are bounded on $L^{2}(0, \infty)$, and $L^{2}(0, \infty) \cap$ $L^{1}(0, \infty)$ is dense in $L^{2}(0, \infty)$. This implies $T \mathcal{H}_{\nu}=\mathcal{H}_{\nu} S$ on $L^{2}(0, \infty)$.

Since $\mathcal{H}_{\mu} \mathcal{C}_{\mu}^{\nu}=T \mathcal{H}_{\nu}$ on $L^{2}(0, \infty)$ by the definition, it follows from the inversion formula $\mathcal{H}_{\mu} \mathcal{H}_{\mu}=I$ that $\mathcal{C}_{\mu}^{\nu}=\mathcal{H}_{\mu} T \mathcal{H}_{\nu}$, and hence the identity $T \mathcal{H}_{\nu}=\mathcal{H}_{\nu} S$ leads to $\mathcal{C}_{\mu}^{\nu}=\mathcal{H}_{\mu} \mathcal{H}_{\nu} S=\mathcal{T}_{\mu}^{\nu} S$ on $L^{2}(0, \infty)$, which completes the proof of the Lemma.

Proof of Theorem 2. (i) Let $\mu \geq-1 / 2$ and $\nu>-1 / 2$. Suppose that $1<p<\infty$. For $f \in L^{p}(0, \infty) \cap L^{2}(0, \infty)$, we infer by the Lemma and Schindler's representation that $\mathcal{C}_{\mu}^{\nu} f(x)=\mathcal{T}_{\mu}^{\nu} S f(x)=T_{\mu, \nu} S f(x)$ for a.e. $x>0$. Thus, it follows from [B](ii) and (3) that

$$
\left\|\mathcal{C}_{\mu}^{\nu} f\right\|_{L^{p}(0, \infty)}=\left\|T_{\mu, \nu} S f\right\|_{L^{p}(0, \infty)} \leq C\|S f\|_{L^{p}(0, \infty)} \leq C\|f\|_{L^{p}(0, \infty)} .
$$

By the standard density argument, we complete the proof of the case $1<$ $p<\infty$ in part (i).

Let us treat the case $p=1$. For $f \in L^{1}(0, \infty) \cap L^{2}(0, \infty)$, by the same reason as above we have $\mathcal{C}_{\mu}^{\nu} f(x)=T_{\mu, \nu} S f(x)$ for a.e. $x>0$. Since $(d / d x) S f(x)=-f(x) / x$ for a.e. $x>0$, it follows that $x|d(S f)(x)|=|f(x)|$, 
and so Theorem 1(i) leads to

$$
\begin{aligned}
\left\|\mathcal{C}_{\mu}^{\nu} f\right\|_{L^{1}(0, \infty)}=\left\|T_{\mu, \nu} S f\right\|_{L^{1}(0, \infty)} & \leq C\left(\|S f\|_{L^{1}(0, \infty)}+\|f\|_{L^{1}(0, \infty)}\right) \\
& \leq C\|f\|_{L^{1}(0, \infty)} .
\end{aligned}
$$

Therefore, $\mathcal{C}_{\mu}^{\nu}$ uniquely extends to a bounded operator on $L^{1}(0, \infty)$.

Let $f \in H^{1}(0, \infty) \cap L^{2}(0, \infty)$. By the Lemma, we have $\mathcal{C}_{\mu}^{\nu} f(x)=\mathcal{T}_{\mu}^{\nu} S f(x)$ for a.e. $x>0$. We know that $S$ is a bounded operator on $H^{1}(0, \infty)$. Thus, if $\mu \geq-1 / 2$ and $\nu>-1 / 2$, then [C](i) implies that

$$
\left\|\mathcal{C}_{\mu}^{\nu} f\right\|_{H^{1}(0, \infty)}=\left\|\mathcal{T}_{\mu}^{\nu} S f\right\|_{H^{1}(0, \infty)} \leq C\|S f\|_{H^{1}(0, \infty)} \leq C\|f\|_{H^{1}(0, \infty)} .
$$

Then the density argument completes the proof of (i).

(ii) Let $\mu \geq-1 / 2$ and $1<p<\infty$. By the Lemma and Schindler's result, in the same way as in the case $\mu \geq-1 / 2, \nu>-1 / 2$ and $1<p<\infty$ of part (i), we see that $\mathcal{C}_{\mu}^{-1 / 2}$ uniquely extends to a bounded operator on $L^{p}(0, \infty)$, and $\left\|\mathcal{C}_{\mu}^{-1 / 2} f\right\|_{L^{p}(0, \infty)} \leq C\|f\|_{H^{p}(0, \infty)}$. For the case $p=1$, let $f \in$ $H^{1}(0, \infty) \cap L^{2}(0, \infty)$. By [C](ii) we have

$$
\left\|\mathcal{C}_{\mu}^{-1 / 2} f\right\|_{L^{1}(0, \infty)}=\left\|\mathcal{T}_{\mu}^{-1 / 2} S f\right\|_{L^{1}(0, \infty)} \leq C\|S f\|_{H^{1}(0, \infty)} \leq C\|f\|_{H^{1}(0, \infty)} .
$$

The density argument allows us to get part (ii) of Theorem 2.

\section{References}

[1] R. Askey, A transplantation theorem for Jacobi series, Illinois J. Math. 13 (1969), 583-590.

[2] R. Askey and S. Wainger, A transplantation theorem between ultraspherical series, ibid. 10 (1966), 322-344.

[3] G. B. Folland, Real Analysis, Modern Techniques and Their Applications, 2nd ed., Wiley, New York, 1999.

[4] J. García-Cuerva and J. L. Rubio de Francia, Weighted Norm Inequalities and Related Topics, North-Holland, Amsterdam, 1985.

[5] C. Georgakis, The Hausdorff mean of a Fourier-Stieltjes transform, Proc. Amer. Math. Soc. 116 (1992), 465-471.

[6] D. V. Giang and F. Móricz, The Cesàro operator is bounded on the Hardy space $H^{1}$, Acta. Sci. Math. (Szeged) 61 (1995), 535-544.

[7] J. L. Gilbert, Maximal theorems for some orthogonal series I, Trans. Amer. Math. Soc. 145 (1969), 495-515.

[8] R. R. Goldberg, Convolutions and general transforms on $L^{p}$, Duke Math. J. 27 (1960), 251-259.

[9] D. L. Guy, Hankel multiplier transforms and weighted p-norms, Trans. Amer. Math. Soc. 95 (1960), 137-189.

[10] G. H. Hardy, Notes on some points in the integral calculus, Messenger Math. 58 (1929), 50-52.

[11] —, On a theorem of Paley and Wiener, Proc. Cambridge Philos. Soc. 33 (1937), $1-5$. 
[12] Y. Kanjin, A transplantation theorem for Laguerre series, Tôhoku Math. J. (2) 43 (1991), 537-555.

[13] - The Hausdorff operators on the real Hardy spaces $H^{p}(\mathbb{R})$, Studia Math. 148 (2001), 37-45.

[14] -, A transplantation theorem for the Hankel transform on the Hardy space, Tôhoku Math. J. 57 (2005), 231-246.

[15] M. Kinukawa and S. Igari, Transformations of conjugate functions, ibid. 13 (1961), $274-280$.

[16] N. N. Lebedev, Special Functions and Their Applications, Dover, New York, 1972.

[17] E. Liflyand and F. Móricz, The Hausdorff operator is bounded on the real Hardy space $H^{1}(\mathbb{R})$, Proc. Amer. Math. Soc. 128 (2000), 1391-1396.

[18] C.-T. Loo, Note on the properties of Fourier coefficients, Amer. J. Math. 71 (1949), 269-282.

[19] A. Miyachi, A transplantation theorem for Jacobi series in weighted Hardy spaces, Adv. Math. 184 (2004), 177-206.

[20] - Transplantation theorem for Jacobi series in weighted Hardy spaces, II, preprint.

[21] F. Móricz, The harmonic Cesàro and Copson operators on the spaces $L^{p}, 1 \leq p \leq$ $\infty, H^{1}$, and BMO, Acta Sci. Math. (Szeged) 65 (1999), 293-310.

[22] B. Muckenhoupt, Transplantation theorems and multipliers for Jacobi series, Mem. Amer. Math. Soc. 356 (1986).

[23] S. Schindler, Explicit integral transform proofs of some transplantation theorems for the Hankel transform, SIAM J. Math. Anal. 4 (1973), 367-384.

[24] A. G. Siskakis, The Cesàro operator is bounded on $H^{1}$, Proc. Amer. Math. Soc. 110 (1990), 461-462.

Mathematics Section

Division of Innovative Technology and Science

Graduate School of Natural Science and Technology

Kanazawa University

Kanazawa 920-1192, Japan

E-mail: kanjin@kenroku.kanazawa-u.ac.jp

Received July 9, 2004

Revised version January 5, 2006 